\title{
Crisis de pánico en un paciente con acné tratado con isotretinoína. Caso clínico
}

\author{
Catalina Poblete $A^{1}$, Viviana Herskovic $M^{2}$, Pedro Eva $C^{2}$. \\ Panic attacks in a patient treated \\ with isotretinoin for acne. \\ Report of one case
}

The use of isotretinoin as a treatment for acne is related to psychiatric syndromes such as psychosis and depression. On the other hand, several drugs have been identified as causing panic attacks. A relationship between dermatologic and psychiatric disease has also been established. We report a 20 year-old male who started to suffer panic attacks after using isotretinoin for acne (Rev Méd Chile 2006; 134: 1565-7).

(Key words: Acne vulgaris; Isotretinoin; Panic)

Recibido el 17 de enero, 2006. Aceptado el 18 de mayo, 2006.

${ }^{1}$ Servicio de Salud Mental Infantil, Hospital Dr. Roberto del Río.

${ }^{2}$ Clínica Psiquiátrica Universitaria, Universidad de Chile, Santiago de Chile.

$\boldsymbol{E}^{\mathrm{s}}$ de conocimiento general que, tanto la piel como el sistema nervioso, derivan embriológicamente de la capa de células ectodérmicas ${ }^{1}$. Por lo tanto, desde su origen existe una relación directa y compleja entre ambos sistemas. En la literatura está descrita una relación recíproca entre enfermedades psiquiátricas y enfermedades dermatológicas ${ }^{2}$. En los pacientes ambulatorios que consultan por enfermedades dermatológicas, se ha estimado una prevalencia de $25 \%$ a $43 \%$ de patología psiquiátrica ${ }^{3}$, siendo las principales comorbilidades descritas los trastornos depresivos, ansiosos y somatomorfos ${ }^{4}$. Se ha estimado también, que pacientes con acné, alopecia, prurito y urticaria, presentarían más patología psiquiátrica, lo que se refleja en baja autoestima, ideación

Correspondencia a: Dra. Viviana Herskovic M. Clínica Psiquiátrica Universitaria. Avda. La Paz 1003. Fono-fax: 2739897. E mail: viviherskovic@gmail.com, suicida, alteraciones del ánimo y en la calidad de vida $^{5-8}$. En particular, los pacientes con acné tendrían un rango de respuestas psicológicas a su enfermedad, entre las que se han descrito baja autoestima y autoconfianza (autoeficacia), ansiedad leve, vergüenza y percepción de ser rechazado socialmente ${ }^{9}$. Se ha relacionado asimismo, la no mejoría de la enfermedad dermatológica con la aparición de la patología psiquiátrica ya descrita ${ }^{5}$.

El acné es una patología dermatológica frecuente entre los adolescentes, con una prevalencia estimada de $80 \%$ a $90 \% 9$. Es importante la búsqueda de tratamientos dermatológicos efectivos para evitar el desarmollo de sintomatología psiquiátrica. Un fármaco utilizado en los casos de acné nodular severo es la isotretinoína, aprobado para este fin por la Food and Drug Administration (FDA) de los Estados Unidos de Norteamérica en $1982^{10}$. La isotretinoína es un retinoide, el isómero del ácido 13-cis-trans-retinoico y derivado de la vitamina $A^{9}$. Su principal metabolito es la 4-oxo-isotretinoína. La vida media de la isotretinoína es de 6 a 36 h y, en administración continua y repetida, 
se establecen concentraciones de estado estable en el transcurso de 5 a 7 días. Varios metabolitos de la isotretinoína se eliminan lentamente del plasma y tienen efecto teratogénico, por lo que se recomienda el uso de anticoncepción por al menos hasta un mes después de la suspensión de isotretinoína ${ }^{11}$.

Entre 1982 y diciembre 2002 el AERS (Sistema de Informe de Eventos Adversos) de la FDA presentaba 3.104 reportes de eventos adversos psiquiátricos atribuidos a isotretinoína, incluyendo 173 suici$\operatorname{dios}^{10}$. No hay evidencia que asegure una relación causal, ni un mecanismo neurobiológico conocido que explique estos síntomas. Sin embargo, sí se conoce la existencia de receptores retinoides distribuidos en el cerebro ${ }^{10}$. Por lo tanto, se postula que las manifestaciones psiquiátricas serían una reacción idiosincrásica a la isotretinoína.

Se ha descrito numerosos fármacos como causantes de crisis de pánico, entre éstos las anfetaminas, buspirona, clobazam, cocaína, fenfluramina, flumacenil, fluoxetina, trazodona, aspartame, fenilefrina, lactato oral, lactato de sodio, oximetazolina, sumatriptan, cotrimoxazol $^{12}$ y como causantes de ansiedad, la yohimbina, ácido lisérgico, cafeína, tabaco, glutamato, L-dopa, corticoides, indometacina, aminofilina, efedina, anorexígenos, marihuana. También han sido relacionados a síntomas de ansiedad algunos déficit vitamínicos, abstinencia de nicotina, opiáceos, alcohol, hipnóticos, ansiolíticos y tricíclicos ${ }^{13}$. Existen estudios de imagenología funcional del cerebro durante crisis de pánico producidas por drogas como lactato de sodio, colecistoquinina 4 , yohimbina y pentagastrina, en los que se ha detectado múltiples diferencias entre sujetos controles y aquellos con crisis de pánico, como asimetría hipocampal, menor perfusión bilateral, hipo e hiperactividad de distintas áreas cerebrales ${ }^{14,15}$.

Los reportes de eventos adversos de la isotretinoína describen su asociación con depresión, episodios psicóticos, intentos de suicidio y suicidio consumado. Hasta el momento, y de acuerdo a la literatura revisada, no existen reportes de crisis de pánico asociadas al uso de isotretinoína. Éste es el motivo de presentar el siguiente caso, el cual sería un primer reporte, según revisión bibliográfica en Medline y Google Scholar hasta diciembre de 2005.

Caso clínico. Paciente de sexo masculino, 20 años, fratría menor de cuatro, estudiante universitario, previamente sano, sin antecedentes de abuso de sustancias ni de tratamiento farmacológico, sin antecedentes familiares de trastomo de pánico. Consultó en dermatología en forma ambulatoria por estar cursando cuadro de acné nodular localizado en tercio inferior de la cara, indicándose isotretinoína (Piplex ${ }^{\circledR}$ ) $20 \mathrm{mg} / \mathrm{d}$ por 30 días. Inició su medicación a mediados de septiembre de 2004, cumpliendo 18 días de tratamiento, el que discontinuó, sin indicación médica, por referir sensación de aumento de nerviosismo» y visión algo borrosa. Siete días tras la suspensión de la isotretinoína, presentó una primera crisis de pánico, caracterizada por sudoración, taquicardia, visión bomosa, sensación subjetiva de mareo, nerviosismo y sofocación, asociada a sensación inminente de muerte. Esta crisis apareció estando acompañado en el interior de un centro comercial, fue de inicio brusco, de aproximadamente 10 min de duración y cedió en forma espontánea. Otra crisis, de iguales características, se repitió pocos días después, estando en la calle. Luego del último episodio, el 12 de octubre de 2004, consultó en Servicio de Urgencia, donde se le suginó control psiquiátrico, en el cual se indicó sertralina 25 $\mathrm{mg} / \mathrm{d}$ y clonazepam 0,5 $\mathrm{mg}$ cada $12 \mathrm{~h}$. Desde esa fecha y hasta la actualidad no ha presentado nuevas crisis de pánico. Durante el período de uso de isotretinoína y del cuadro descrito no presentó síntomas depresivos, psicóticos ni ideación suicida.

El paciente tiene como antecedente haber presentado en la infancia, en forma repetida, síntomas de la esfera ansiosa que no cumplen criterios diagnósticos para crisis ni trastorno de pánico. Según lo informado por él, aquel cuadro no fue formalmente diagnosticado, siendo tratado con sulpiride y ácido gamabutírico. El trastorno cedió y el paciente suspendió el tratamiento, sin volver a presentar síntomas.

\section{DisCUSIÓN}

La literatura informa sobre una probable relación entre depresión, psicosis, suicidio y el uso de isotretinoína, pero esos reportes no han logrado demostrar una causalidad directa ${ }^{16,17}$. Ellos advierten, sin embargo, que puede existir esta asociación, por lo que se debe evaluar la presencia de síntomas psiquiátricos en los pacientes tratados con este fármaco. En el caso descrito no se pudo confirmar una relación causal fármaco-síntomas, dado que el paciente rechazó la reinstalación del medicamento 
una vez suspendido. Es aventurado asegurar una relación causal porque los síntomas más intensos se presentaron sólo después de la suspensión del fármaco. Como el único período en que el paciente presentó síntomas de pánico fue cuando ya había usado el medicamento, y como éste tiene una vida media prolongada, se puede sospechar que estos síntomas se hayan debido a su uso. De esta manera, se sugiere una posible relación causal, que no se puede afirmar categónicamente.

Chee Hong ${ }^{18}$ comparó los síntomas psiquiátricos en pacientes con acné tratados con isotretinoína

\section{REFERENCIAS}

1. SADIER TW. Capítulo 5 Tercera a octava semana: el período embrionario. En: Sadler TW, Langman. Embriología médica con orientación clínica. Madrid: 8a edición, Editorial Médica Panamericana, 2001; pág. 81-88.

2. Gupta MA, Gupta AK. Psychiatric and Psychological Comorbidity in Patients with Dermatologic Disorders: Epidemiology and Management. Am J Clin Dermatol 2003; 12: 833-42.

3. Picardi A, Abeni D, Renzi C, Braga M, Meichi CF, Pasquin $P$. Treatment outcome and incidence of psychiatric disorders in dermatological outpatients. J Eur Acad Dermatol Venereol 2003; 17: 155-9.

4. AкTan S, Ozmen E, SanL B. Psychiatric disorders in patients attending a dermatology outpatient clinic. Dermatology 1998; 197: 230-4.

5. MiLaRd L Dermatological practice and psychiatry. $\mathrm{Br}$ J Dermatol 2000; 5: 920.

6. GINSBURG IH. The psychological impact of skin disease: an overview. Dermatol Clinics 1996; 14: 47384.

7. GuPTA MA, GuPTA AK. Isotretinoin use and reports of sustained dreaming. Br J Dermatol 2001; 4: 920.

8. KeULe SC, GawKrodger DJ. The psychological and emotional impact of acne and the effects of treatment with isotretinoin. Br J Dermatol 1999; 140: 273-82.

9. Jick SS, KrEMERS HM, VasilaKIs-ScaramozZa C. Isotretinoin Use and Risk of Depression, Psychotic Symptoms, Suicide, and Attempted Suicide. Arch Dermatol 2000; 136: 1231-6.

10. HuL PR, D'ArCY C. Isotretinoin Use and Subsequent Depression and Suicide: Presenting the Evidence. Am J Clin Dermatol 2003; 7: 493-505.

11. Marcus R, Coulston AM. Capítulo 64 Vitaminas versus antibióticos y tratamiento tópico, no encontrando diferencias estadísticamente significativas entre ambos grupos en cuanto a aparición de psicopatología. Dado que los artículos demuestran que la presencia de acné en los adolescentes genera síntomas psiquiátricos, que la isotretinoína es una altemativa eficaz de tratamiento, que ésta ha demostrado disminuir la ansiedad en un grupo de pacientes con acné $^{19}$ y que los efectos adversos psiquiátricos son escasos e idiosincrásicos, se justificańa su prescripción ya que los beneficios superańan a los niesgos.

Liposolubles Vitaminas A, K y E. En: Hardman JG, Limbir LE ed. Goodman y Gilman. Las bases farmacológicas de la terapéutica, Volumen 2. México: 10aㅡ edición, Editorial Mc Graw Hill, 2003; pág. 1797-802.

12. Bazire S. Capítulo 5 Trastomos psiquiátricos inducidos por fármacos u otras sustancias. En: Bazire S. Formulario de fármacos psicotrópicos. Reino Unido: Quay Books, 2000.

13. VAuEjo J. Capítulo 25 Trastomos de angustia. En: Vallejo Ruiloba J. Introducción a la psicopatología y psiquiatría. Barcelona: 5a edición, Editorial Masson, 2002; pág. 379-394.

14. Boshuisen ML, Ter Horst GJ, PaAns A, Reinders AA, Den BoER JA. rCBF differences between panic disorder patients and control subjects during anticipatory anxiety and rest. Biol Psychiatry 2002; 52: 126-35.

15. Bonne O, Drevets WC, NeumeISTER A, Charney DS. Chapter 47 Neurobiology of anxiety disorders. En: Schatzberg A, Nemeroff C, ed. Textbook of Psychopharmacology. Arlington VA, USA: Tencera Edición, The American Psychiatry Publishing, 2004.

16. ENDERS SJ, ENDERS JM. Isotretinoin and Psychiatric Illness in Adolescents and Young Adults. Ann Pharmacother 2003; 7: 1124-7.

17. CheE Hong N, Schwetrzer I. The association between depression and isotretinoin use in acne. Austr New Zeal J Psychiatry 2003; 1: 78-84.

18. Chee Hong N, Tam M, Cei E, Tate B, Schweitzer I. Prospective study of depressive symptoms and quality of life in acne vulgaris patients treated with isotretinoin compared to antibiotic and topical therapy. Autralasian J Dermatol 2002; 4: 262-8.

19. Ferahbas A, Turan MT, Esel E, Utas S, Kutkugun C, Kшc Gur C. A pilot study evaluating anxiety and depressive scores in acne patients treated with isotretinoin. J Dermatolog Treat 2004; 15: 153-7. 